\title{
Análisis de contenido informatizado para las aproximaciones al concepto de objetividad de los profesionales de la información de la cultura periodística de España
}

\section{Computerized content analysis to approach the concept of objectivity maintained by professional journalists in Spain}

\author{
Daniel Barredo Ibáñez \\ daniel.barredo@udla.edu.ec \\ Universidad de las Américas, Ecuador \\ Martín Oller Alonso \\ oller.martin@udla.edu.ec \\ Universidad de las Américas, Ecuador \\ Sergio Hernández \\ sehernandez@uv.mx \\ Universidad Veracruzana, México
}

doi: $\underline{\text { http://dx.doi.org/10.15359/abra.35-50.2 }}$

\begin{abstract}
Resumen: Este artículo analiza la idea de objetividad que tienen los periodistas españoles (entiéndase mujeres y hombres que ejercen la profesión de periodista), porque este es uno de los principales conceptos que estructuran los valores y las ideas de la cultura periodística de un país. En primer término los autores de este proyecto desarrollaron un estudio cualitativo integrado por una muestra representativa de 19 informadores de origen español procedentes de diferentes organizaciones o medios de comunicación (prensa, radio, televisión y medios digitales). La metodología utilizada -una entrevista en profundidad- ofreció la posibilidad de recabar información sobre asuntos concretos, entre los cuales sobresalió la opinión individual sobre la objetividad. Pero en segundo término se analizaron las respuestas mediante un análisis de contenido informatizado de las asociaciones léxicas establecidas entre los términos más frecuentes. A través de la triangulación de ambas metodologías (cualitativa y cuantitativa) mostramos como resultados los elementos que vertebran el concepto de objetividad para la comunidad periodística española entrevistada; además, la descripción instrumental -con una combinación de técnicas tan novedosas- se ofrece como otro de los grandes hallazgos de este trabajo.
\end{abstract}

Palabras clave: Objetividad, metodología cualitativa, cultura periodística española, análisis de contenido informatizado.

Daniel Barredo, Martín Oller y Sergio Hernández

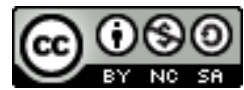

Esta obra está bajo licencia

Creative Commons Reconocimiento-NoComercial-Compartirlgual 4.0 Internacional License 


\begin{abstract}
The idea of objectivity that Spanish journalists have (i.e. women and men who work as journalists) is analyzed in this paper since this is one of the main concepts that shape the values and ideas of the journalism culture in a country. First, a qualitative study was conducted with a representative sample of 19 journalists of Spanish origin from different organizations or media (press, radio, television and digital media). The methodology used, the in depth interview, offered the possibility to gather information on specific issues, particularly the individual perception of objectivity. Second, responses were studied using a computerized content analysis of lexical associations between the most frequent terms. Through the triangulation of both methods (qualitative and quantitative) the authors showed, as results, the elements that form the backbone of the concept of objectivity for the interviewed Spanish journalistic community. In addition, another major finding resulting from this research was the instrumental description, which included a combination of very novel techniques.
\end{abstract}

Keywords: objectivity, qualitative methodology, Spanish journalism culture, computerized content analysis.

Fecha de recepción: 25-11-2014 Fechas de reenvíos: 19-03-2015/23-03-2015 Fecha de aceptación: 14-04-2015 Fecha de publicación: 19-05-2015.

\title{
Introducción
}

El concepto de objetividad se encuentra profundamente arraigado en la comprensión periodística con la que los profesionales tienden a estructurar la realidad. Esta noción que actúa como un filtro se entiende como la adopción de posturas dinámicas, lejos de clasificaciones políticas 0 personales. Los periodistas, como intermediarios del colectivo, forman parte fundamental del proceso democrático (Deuze, 2002), por lo que sus posicionamientos implican mucho más que simples cuestiones profesionales. En un plano ideal, los informadores gestionan una representación conformada por las distintas tendencias sociológicas.

La idea de objetividad está presente en el trabajo diario de los periodistas. Tanto desde un punto de vista filosófico, como con base en unas convicciones éticas o en la elaboración y ejecución de un método de trabajo. Existen detractores que niegan la posibilidad de alcanzarla "desde el momento mismo en que en las salas de redacción [...] se empieza a tomar posición en torno a tener que decidir entre decenas de historias" (Saad, 2012) y defensores que ven en ella la base de una profesión honesta y "como medio de justificación de la profesionalización del periodismo" (Oller, Chavero, Ortega y Pullas, 2014, p. 259). Por lo que, cómo es comprendido el concepto de objetividad y la importancia de esta depende del contexto cultural de los periodistas (Donsbach y Klett, 1993, p. 57-58). De forma que una correcta comprensión de la idea de objetividad que los periodistas tienen, nos ayudará a una mejor comprensión de la cultura periodística española.

Este estudio analiza la idea de objetividad que tienen 19 periodistas españoles activos pertenecientes a medios de comunicación dentro del territorio nacional. Para ello, se realizó un trabajo empírico basado en una técnica cualitativa (entrevistas en profundidad). El análisis informatizado de los resultados se ha llevado a cabo a partir de las asociaciones léxicas que nos

Daniel Barredo, Martín Oller y Sergio Hernández

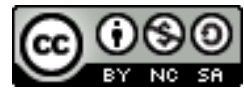

Esta obra está bajo licencia

Creative Commons Reconocimiento-NoComercial-Compartirlgual 4.0 Internacional License 
permitió establecer la frecuencia de aparición de ciertos términos en las respuestas de las personas entrevistadas. De forma que este grado de relación de los términos contiguos nos ayudó a determinar el nivel de relevancia y significatividad del concepto de objetividad dentro del discurso argumentativo de los periodistas.

El propósito de este proyecto es dar respuesta a las siguientes preguntas: 1. ¿qué entienden los periodistas españoles por objetividad como ideal?; 2 . ¿qué métodos objetivos priorizan los periodistas en España?; y 3. ¿qué influencia ejercen los factores contextuales en la idea de objetividad que tienen los periodistas?

\section{El concepto de objetividad}

La objetividad obedece más a una estructura colectiva que a un mundo subjetivo. De forma que los fenómenos no llevan impresos en sí mismos el carácter de mensaje o no mensaje. Estos son neutrales y los sujetos los experimentan de forma bipolar con una realidad objetiva y una subjetiva (Valbuena de la Fuente, 1997, p. 327).

En los años veinte del siglo pasado Lippman (1920) planteó un periodismo basado en lo real del mundo. En donde se aspira a un cambio serio y una democracia basada en los hechos objetivos (Donsbach y Klett, 1993, p. 55). A partir de este momento, el método objetivo se instauró dentro del periodismo como base filosófica y como método general de trabajo (Schudson, 2001, p. 162).

Esta corriente basada en la objetividad perduró hasta los años 70 y 80 del siglo pasado en el que la relación de los periodistas y la objetividad evolucionó y situó los hechos en un paradigma diferente dentro del proceso comunicativo. Lo que marcó el comienzo de una corriente crítica respecto a la objetividad y a su necesaria implantación en el periodismo. De modo que, como afirmó Canel (1999), un gran número de investigaciones llevadas a cabo en los últimos años del siglo pasado asumieron que las noticias son la representación de la realidad que hacen los periodistas. Corriente que está teniendo su continuación en las dos primeras décadas del siglo XXI en el que "las propias voces de los periodistas ayudan a comprender la complejidad del escenario en que hoy se desarrolla la profesión, que obliga a refinar el debate y reemplazar la antigua objetividad por conceptos más matizados" (San Martín, 2008, p. 79).

De modo que a través del análisis de contenido de las entrevistas realizadas a 19 periodistas en España, pretendemos mostrar la idea de objetividad que estos tienen dentro de su contexto real de trabajo. Tanto de un punto de vista filosófico (subjetivismo, realidad, veracidad o verdad), en donde se hace referencia real a la realidad representada (Hackett, 2008), como de su puesta en escena al ser utilizada como método estandarizado de trabajo, o la que Donsbach (1990, p. 18) describió como la "noción funcional de la objetividad".

Daniel Barredo, Martín Oller y Sergio Hernández

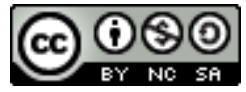

Esta obra está bajo licencia 


\section{Metodología}

\section{en España. \\ Estudio cualitativo: análisis de la objetividad dentro de la cultura periodística}

Esta investigación cualitativa ha sido llevada a cabo a partir de un estudio de campo basado en la realización de 19 entrevistas en profundidad a periodistas españoles en activo dentro de algunos de los principales medios de comunicación en España (RTVE, Cadena SER, Público y El Mundo) entre enero de 2009 y julio de 2011. Estos son denominados "medios de calidad" por poseer una línea editorial, una influencia concreta, por formar opinión pública y ser referentes de otros medios (Jarren y Vogel, 2008; Oller y Meier, 2012). La selección de la muestra se hizo a través de un muestreo por cuotas, a partir de parámetros que proporcionaran la mayor representatividad de los medios de comunicación en España.

Los autores se basaron en la teoría deductiva de Mayring (2002) y las dimensiones estructurales que debe contener un análisis cualitativo. La herramienta utilizada en el estudio cualitativo ha sido un cuestionario estructurado en dos áreas de investigación: la objetividad como ideal y la objetividad como método. Cada una de estas áreas contaba con un número determinado de preguntas que se realizaron a los periodistas entrevistados. La primera área basada en el concepto de objetividad como ideal estaba dividida a su vez en dos subcategorías o dimensiones: "correspondencia-subjetividad". Para ello se utilizó la clasificación llevada a cabo por Hanitzsch (2007) en el proyecto Worlds of Journalism ${ }^{1}$. La segunda área, la encargada de analizar el concepto de objetividad como método, se basó en las cinco estrategias de Tuchman (1978, p. 1) distintos puntos de vista; 2) fidelidad a los hechos; 3) citas; 4) la pirámide invertida; y 5) la separación de noticia y opinión. Aunque, también se tuvieron en cuenta otros métodos objetivos que los periodistas expusieron en sus entrevistas.

Además, el cuestionario contaba con una serie de preguntas de tipo sociodemográfico a partir de las cuales los autores pudieron conocer la situación profesional, contractual y el perfil del periodista.

\section{Análisis de las asociaciones léxicas.}

En el análisis de las asociaciones léxicas (realizado en este artículo a través del software Catpac) cada palabra estructural es tratada como una neurona dentro de una matriz de datos, por consiguiente se determina la frecuencia de aparición de los términos de un texto, así como su grado de relación con los términos contiguos y gestiona toda esa información en una "red neuronal artificial" (Woelfel, 1998, p. 11). Cada palabra estructural ${ }^{2}$ es tratada como una neurona dentro de una matriz de datos (Chen, Evans, Battleson, Zubrow \& Woelfel, 2011). El resultado es un procedimiento cuantitativo, preciso, objetivo y fácilmente validable

\footnotetext{
${ }^{1}$ Consultado 23/03/2015: http://www.worldsofjournalism.org/

${ }^{2}$ Que son denominadas posteriormente palabras-tema.
}

Daniel Barredo, Martín Oller y Sergio Hernández

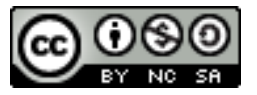


por otros investigadores (Alexa, 1997; Popping, 2000; Tian y Stewart, 2005). Al combinar el análisis de contenido informatizado con otros procedimientos -como la entrevista en profundidad-, consideramos que hay dos condiciones previas sin las cuales no puede efectuarse un trabajo riguroso de este tipo:

1) Tiene que ser un análisis focalizado. Se debe limitar la heterogeneidad en la muestra (es decir, la injerencia de bloques temáticos diversos), con el fin de obtener, en la medida de lo posible, un bloque léxico homogéneo. En nuestro análisis, por ejemplo, hemos fracturado la entrevista en bloques de sentido (para lo cual solo planteamos preguntas alrededor de la objetividad), los cuales examinaremos separadamente.

2) Tiene que ser un análisis adaptado. El objetivo no es construir un análisis estandarizado, el cual podría restringir las posibilidades reales del trabajo en términos de profundidad. Un ordenador, como asegura Diefenbach (2001), no puede actuar aisladamente. De la misma manera en el análisis de contenido informatizado el investigador ha de establecer unas pautas adaptadas al tipo de contexto por evaluar, algo que se ha desarrollado en algunos estudios antecedentes (Barredo, 2013; Barredo y Oller, 2012a, 2012b; Buenaventura, Barredo y Oller, 2012).

\section{Pasos procedimentales.}

En total hemos examinado las siguientes palabras y caracteres (sin espacios):

Tabla1.

Cantidad de palabras y caracteres de las respuestas sobre objetividad

\begin{tabular}{cc}
\hline Palabras analizadas & Caracteres sin espacios \\
\hline 822 & 63.242 \\
\hline
\end{tabular}

Fuente: elaboración propia

Hemos escogido el método de Ward (basado en un algoritmo de repetición de \pm 1 palabra), porque este es el procedimiento recomendado por el desarrollador de Catpac (Woelfel, 1998). De la misma manera, hemos delimitado la red neuronal a los 40 términos claves, por un lado porque esta es una pauta derivada del manual de instrucciones del software (Woelfel, 1998), pero principalmente -como en el caso de los neologismos en los medios de comunicación, según aconseja Hernández (2006)-, hemos exigido un amplio porcentaje de repeticiones antes de considerar una palabra como definitoria de las tendencias grupales. Por el otro lado y para organizar el análisis, hemos emprendido algunos pasos procedimentales mediante exclusiones y recodificaciones.

Daniel Barredo, Martín Oller y Sergio Hernández

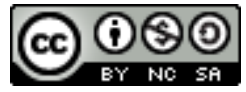

Esta obra está bajo licencia 


\section{Exclusiones.}

En un análisis de asociaciones léxicas no interesa visualizar todo el patrimonio terminológico de una unidad determinada; el interés principal de este tipo de trabajos, más bien, es localizar e identificar las conocidas como palabras-tema, esos términos que configuran las claves temáticas de un texto. En este proyecto partimos de la premisa de la heterogeneidad estructural: la muestra está conformada por 19 entrevistas, las cuales constituyen 19 puntos de vista respectivos alrededor de un concepto (objetividad). Por ello hemos restringido las dimensiones de las palabras-tema a través de las siguientes exclusiones:

Sustantivos poco relevantes para el análisis: son aquellos que introducen puntos de vista difuminados. Se han excluido: cosa, cosas, momento, punto, puntos, ejemplo, hora, día, elementos, años.

Adjetivos. Aluden a perspectivas individuales, no estructurales. Se han excluido: varias, fundamental, diferente, contado, importante, claro, final, muchos, máximo, posible, imposible, fácil, difícil.

Adverbios. Porque sus nociones se activan en función del contexto. Se han excluido: absolutamente, claramente, ahora.

Todos los verbos. En este trabajo hemos decidido excluir todos los verbos en la validación de las asociaciones, ya que buscamos consolidar una conceptualización estructural. Se adjunta a continuación la lista de esos verbos excluidos: dar, decir, hacer, pasar, ver, poder, gustar, contar, creer, depender, escribir, existir, formar, gustar, intentar, buscar, saber, faltar, leer, procurar, dejar, poner; asimismo se han filtrado los verbos modales: querer, poder, deber, tener; y por último los verbos más frecuentes del español: ser, estar, parecer, tener y haber.

\section{Recodificaciones.}

Únicamente se ha efectuado una recodificación: se han agrupado los dos términos pirámide invertida bajo la etiqueta PIRÁMIDEINVERTIDA, porque ambos se refieren a un tipo de estructuración informativa de las noticias en el periodismo.

\section{Resultados}

Al analizar las 40 palabras-tema más repetidas por los 19 periodistas entrevistados, advertimos la vasta cantidad de términos circunscritos al campo semántico del periodismo. Son vocablos que aluden a géneros periodísticos, a formas de componer o a elementos que integran la información. 
Tabla 2.

Cuarenta palabras-tema más repetidas por las y los periodistas en las respuestas sobre objetividad

\begin{tabular}{|c|c|c|}
\hline Palabras & & \\
\hline INFORMACIÓN & 93 & 10 \\
\hline OPINIÓN & 63 & 6,8 \\
\hline OBJETIVIDAD & 62 & 6,7 \\
\hline NOTICIA & 52 & 5,6 \\
\hline PERIÓDICO & 43 & 4,6 \\
\hline GENTE & 39 & 4,2 \\
\hline OBJETIVO & 39 & 4,2 \\
\hline FUENTES & 35 & 3,8 \\
\hline PERIODISTA & 34 & 3,7 \\
\hline FUENTE & 33 & 3,6 \\
\hline VERDAD & 29 & 3,1 \\
\hline MUNDO & 27 & 2,9 \\
\hline LECTOR & 26 & 2,8 \\
\hline PIRÁMIDE INVERTIDA & 23 & 2,5 \\
\hline TRABAJO & 23 & 2,5 \\
\hline PERSONA & 21 & 2,3 \\
\hline TEMA & 20 & 2,2 \\
\hline PERIODISMO & 19 & 2,0 \\
\hline PERIODISTAS & 18 & 1,9 \\
\hline PERIÓDICOS & 17 & 1,8 \\
\hline MEDIO & 15 & 1,6 \\
\hline MEDIOS & 15 & 1,6 \\
\hline ARTÍCULO & 14 & 1,4 \\
\hline SENTIDO & 14 & 1,5 \\
\hline DATOS & 13 & 1,4 \\
\hline PERSONAS & 12 & 1,3 \\
\hline HECHOS & 11 & 1,2 \\
\hline INFORMACIONES & 11 & 1,2 \\
\hline ENFOQUE & 10 & 1,1 \\
\hline RUMOR & 10 & 1,1 \\
\hline RUMORES & 10 & 1,1 \\
\hline CONCEPTO & 9 & 1,0 \\
\hline ESTRUCTURA & 9 & 1,0 \\
\hline INTENCIÓN & 9 & 1,0 \\
\hline NOTICIAS & 9 & 1,0 \\
\hline PRINCIPIO & 9 & 1,0 \\
\hline REPORTAJE & 9 & 1,0 \\
\hline ARTÍCULOS & 8 & 0,9 \\
\hline ESTILO & 8 & 0,9 \\
\hline LECTORES & 8 & 0,9 \\
\hline
\end{tabular}

Fuente: elaboración propia

De las palabras-tema anteriores únicamente seis términos (gente, objetivo, mundo, trabajo, persona, personas) se refieren a realidades que podrían considerarse relativamente al margen de la actividad profesional de los periodistas, las cuales describiremos en las siguientes páginas. Las otras treinta y cuatro palabras-tema restantes presentan una gran estabilidad léxica, con un fuerte relacionamiento con el ámbito profesional del periodismo. Es este un rasgo que redunda en la calidad de la entrevista; los usos grupales limitan en buena medida la

Daniel Barredo, Martín Oller y Sergio Hernández 
injerencia de términos no vinculados con la reflexión sobre el periodismo, porque es esta una entrevista especializada y centrada en un fenómeno dado.

Pero, además de desmontar terminológicamente los conceptos que asientan la noción de la objetividad entre los informadores, el otro gran interés de este análisis es determinar las asociaciones que se establecen entre esos mismos términos. En el siguiente mapa perceptual notamos que el grupo de entrevistados tiende a asociar esos cuarenta términos en dos corrientes dispersas según el posicionamiento general de las asociaciones. El grado de cercanía entre las etiquetas terminológicas significa, asimismo, el grado de cercanía en que esas palabras suelen aparecer en las respuestas evaluadas ${ }^{3}$ :

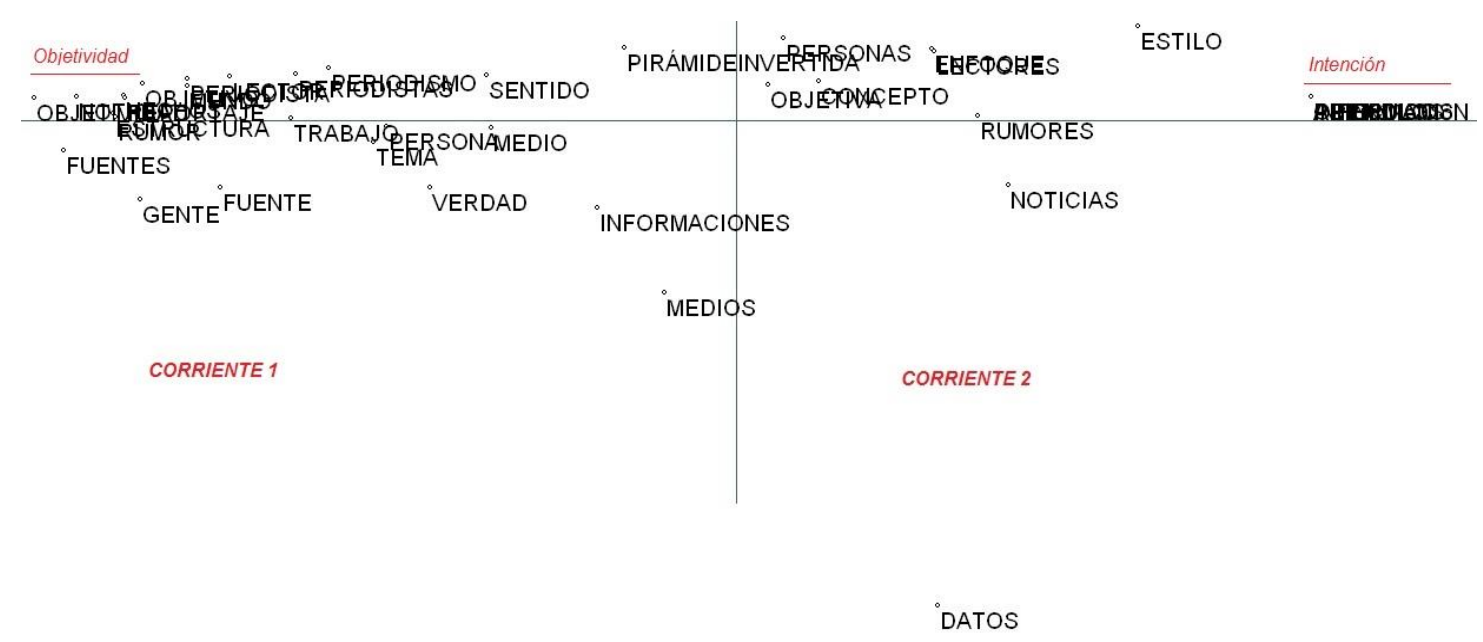

Figura 3. Mapa situacional estructural de las asociaciones léxicas de las cuarenta palabras-tema de la objetividad Fuente: ThoughView. Elaboración propia

La dispersión terminológica depende fundamentalmente de la heterogeneidad de la muestra; en otros trabajos (Barredo y Oller, 2012a; 2012b) contamos con muestras relativamente homogéneas, por lo que anotamos la presencia de bloques de sentido no tan diseminados como los que se aprecian en la figura 3. Si atendemos al mapa estructural, advertimos dos tendencias de proximidad: la corriente primera, donde parece que el término clave es la palabra que origina las preguntas de la encuesta (objetividad), y la corriente segunda, que contiene un grupo de palabras-tema muy frecuentemente asociadas alrededor de un término abstracto (intención), aunque su solapamiento abrupto indica que se trata de una

\footnotetext{
${ }^{3}$ Algunas de las palabras-tema se solapan porque tienden a aparecer juntas muy frecuentemente. A pesar de las dificultades de visualización de algunos términos, subrayamos que este mapa situacional solo tiene un valor ilustrativo estructural. En las páginas siguientes describiremos cada uno de los conjuntos representados.
}

Daniel Barredo, Martín Oller y Sergio Hernández

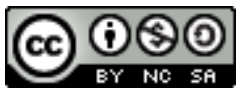

Esta obra está bajo licencia 
microtendencia y no de una preferencia grupal ${ }^{4}$. La distribución léxica entre esas dos corrientes no es homogénea, sino que depende esencialmente de factores significativos y contextuales.

El siguiente mapa perceptual aumenta la visualización de las 16 palabras-tema (personas, concepto, lectores, enfoque, rumores, noticias, principio, datos, estilo, artículo, artículos, información, intención, opinión, periódico, periódicos), que integran la casuística de la corriente $2^{\underline{5}}$.

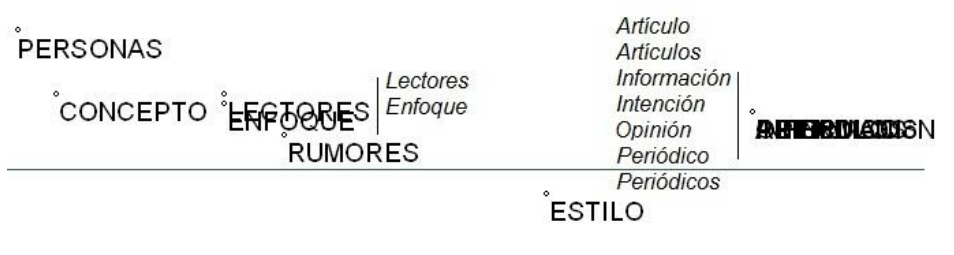

¿NOTICIAS

CORRIENTE 2

PRINCIPIO

DATOS

Figura 1. La corriente 2: microtendencias sobre objetividad. Fuente: ThoughView / elaboración propia.

En un plano general hemos apreciado la elevada cantidad de palabras-tema que aluden a los receptores: persona, personas, gente, lector, lectores. Pero la asociación léxica más interesante, según el mapa anterior, se produce entre el término lectores y el término enfoque: las audiencias, según se filtra en los términos más repetidos por los informadores, determinan la construcción de la perspectiva.

En el grupo sincopado -situado en la parte derecha del mapa- localizamos una asociación entre intención y dos términos clave en el argot periodístico: información y opinión. Aspecto que expone de manifiesto el papel de la subjetividad de la información o del individuo, el cual se sitúa en un

\footnotetext{
${ }^{4}$ Los mapas perceptuales agrupan visualmente unos términos, aunque para una mayor precisión se aconseja complementar estas herramientas con tablas objetivas como la que hemos ofrecido en la figura 2.

${ }^{5}$ En el anexo 1 ofrecemos el dendrograma del que se han extraído las relaciones del mapa.
}

Daniel Barredo, Martín Oller y Sergio Hernández

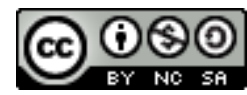

Esta obra está bajo licencia 
punto intermedio entre la objetividad y la subjetividad. De forma que se puede evidenciar la importancia que para los periodistas posee la relación de opinión e información. Aspecto al que hacía referencia Tuchman (1978) en su quinta estrategia. Por lo que se puede deducir que en el periodismo español en general, y en las redacciones de los medios de comunicación analizados en particular, en un primer momento la separación de la noticia y la opinión se produce a nivel formal. Sobre todo en el caso de los medios escritos estudiados donde en la primera sección del periódico aparecen los artículos de opinión y los editoriales.

Dentro de este bloque comprobamos la hegemonía textual, aún prevaleciente en los informadores entrevistados (el $47 \%$ de los encuestados procede de medios de comunicación escritos): los miembros del grupo citan casi cinco veces más a la noticia que a cualquier otro género (indicativo de la importancia concedida a este formato informativo) ${ }^{\underline{6}}$, pero entre los géneros más frecuentes encontramos el artículo (con su variante en plural) ${ }^{\underline{7}}$ y el reportaje. Asimismo, entre los medios más citados, hallamos -incluso en su variante plural-, el que designa al periódico, por encima de los medios audiovisuales o de los cibermedios. Pero los datos más interesantes proceden de la corriente 1, la cual aglutina a 24 de las 40 palabras tema: objetividad, noticia, fuentes, objetivo, hechos, reportaje, estructura, rumor, gente, periodista, mundo, lector, fuente, periodismo, periodistas, trabajo, persona, tema, verdad, sentido, medio, pirámide invertida, informaciones, medios ${ }^{\underline{8}}$ :

CORRIENTE 1

PIRÁMIDEINVERTIDA

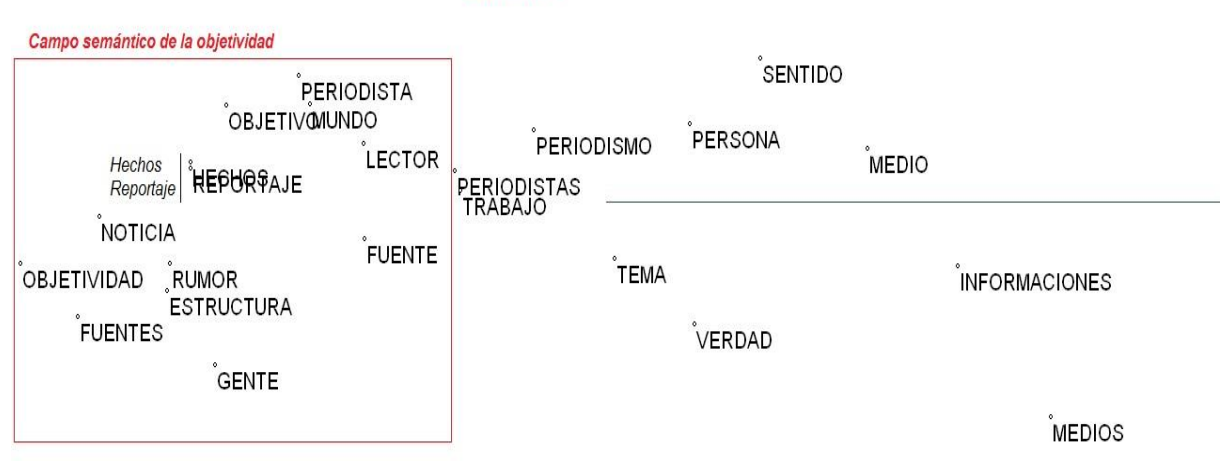

Figura 2. Corriente 1: macrotendencias sobre objetividad. Fuente: ThoughView / elaboración propia

${ }_{7}^{6}$ Las frecuencias de los términos se encuentran en la figura 2.

${ }^{7}$ No hemos recodificado las variantes en plural porque creemos que constituyen términos diferenciados, los cuales tienden a asociarse libremente con otras palabras-tema del mapa.

${ }^{8}$ Para un mayor entendimiento de las relaciones que se establecen en el mapa siguiente, se recomienda visitar el anexo 1 de este artículo.

Daniel Barredo, Martín Oller y Sergio Hernández

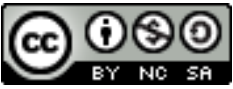

Esta obra está bajo licencia 
Es ese bloque, rotulado en el mapa anterior, el que conforma el campo semántico de la objetividad: la asociación más importante o de primer nivel que se establece entre las 40 palabras-tema, está constituida por objetividad y fuentes. En el caso de los periodistas españoles, las ideas que manejan con respecto a los métodos objetivos utilizados giran alrededor del necesario contraste a partir de la presentación de varias fuentes.

Por lo tanto, para el grupo entrevistado, ser objetivo depende del tipo de fuentes informativas utilizadas, pero es que el género que tiende a asociarse con ese concepto es -un escalón asociativo más abajo- el de la noticia. Recordemos, por ejemplo, la tendencia de posicionar en el mismo contexto -en el otro extremo del mapa, según la figura 3-, intención con información y opinión, entre otras.

A medida que se amplía el radio asociativo, se consolidan nuevas relaciones terminológicas (cada vez más distanciadas) que enriquecen el campo semántico de la objetividad: los informadores mencionan el término estructura, es decir, aluden a la necesidad de organizar las fuentes mediante un procedimiento; también aparece la palabra rumor -y en la otra corriente su variante en plural-, como una antítesis conceptual. Lo que nos permite comprobar como los periodistas españoles admiten, en cierta medida y con reservas, que los rumores tienen su espacio dentro del periodismo y que existe una parte del periodismo, o cierto periodismo, que los tiene presentes como herramientas de trabajo.

Otra sugerente asociación es la que se fija entre hechos y reportaje; resulta coherente en este marco sobre objetividad que los dos géneros informativos aludidos sean, precisamente, el reportaje y sobre todo la noticia. Pero esa mención a los hechos (a la que hace referencia Tuchman (1978) en su segunda estrategia), junto a otras constantes (fuentes, fuente), subrayan la gran importancia que los informadores conceden a las voces con las que fundamentan sus aportaciones. En este primer bloque destaca, asimismo, la entrada de un vocabulario (mundo, lector, gente), el cual enumera a realidades exteriores al periodismo, y señala la importancia otorgada por el grupo a la fiscalización externa para validar la objetividad.

Al margen de estas asociaciones, estrechamente vinculadas a las preguntas de investigación, el resto del mapa contiene nociones más difuminadas; resulta llamativa la exclusión de la pirámide invertida del núcleo central sobre objetividad (lo que nos da a entender la poca relevancia otorgada por los periodistas a esta estructura informativa a la hora de desarrollar sus informaciones, el cual ha sido establecido como un método objetivo más teórico, aprendido en las universidades, que práctico y utilizado de forma cotidiana en las redacciones). De igual modo ocurre con el concepto de verdad, un concepto que concentra un matiz filosófico y que es considerado como poco funcional. Lo que muestra que los periodistas españoles entrevistados son bastante escépticos a la hora de plantear el concepto de objetividad como ideal filosófico dentro del periodismo, una evidencia de la idea práctica que los periodistas entrevistados poseen del concepto de objetividad alejada de su carácter filosófico y centrada en su aplicación y en el uso concreto de las cinco estrategias de la objetividad como método descritas por Tuchman (1978, p. 196).

Daniel Barredo, Martín Oller y Sergio Hernández

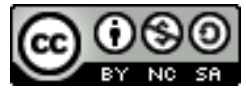

Esta obra está bajo licencia 
Otro hecho importante en esta representación se encuentra en las palabras-tema que hacen referencia al trabajo y al medio. Ninguna de ellas se encuentra dentro del marco referencial de la objetividad. Lo que nos muestra que la influencia del medio en sus concepciones individuales sobre objetividad no aparece como representativa. Además, se observa como la presión ejercida por el medio se encuentra dentro de unos parámetros más que aceptables y que los periodistas cuentan con bastante libertad e independencia. Característica común al resto de culturas periodísticas occidentales debido a sus sistemas políticos basados en la democracia.

\section{Discusión y conclusiones}

A partir de este análisis, los autores han intentado profundizar en el estudio del concepto de objetividad dentro de la cultura periodística española. Concretamente, en el estudio de la idea de objetividad que los periodistas tienen a nivel individual, lo que puede establecer las influencias contextuales que, en cierta medida, determinan el trabajo profesional y el rol ejercido por la objetividad en las redacciones españolas.

La aparición de nuevos instrumentos de medición, como Catpac, impulsa el desarrollo de nuevos procedimientos analíticos o de la complementación -como en este trabajo- de los ya existentes. A través de una perspectiva cuantitativa y objetiva hemos mostrado la fiabilidad de las entrevistas (de carácter cualitativo) realizadas a 19 informadores españoles; se ha enseñado la estabilidad de las respuestas dadas sobre un concepto, así como el intento grupal por establecer una reflexión sobre el periodismo y, en concreto, sobre un fenómeno tan complicado de delimitar como es el de la objetividad.

Para los periodistas entrevistados ser objetivo depende en buena medida del tipo de fuentes con las que se construye la información; ha sido esta la asociación de primer nivel que hemos localizado. En el núcleo de vinculaciones terminológicas sobre la objetividad, encontramos dos géneros predominantes, cuya información tenderá a ser más objetiva (noticia y reportaje), en tanto que las menciones frecuentes a realidades externas -como mundo, lector, gente-, resaltan el ejercicio de transparencia y de verificación social al que se someten o han de someterse los informadores. El periodismo se entiende como un vehículo de doble sentido: una actividad profesional retroalimentada, para ser objetiva, por las audiencias. Pero son las audiencias las que -como hemos mostrado en la corriente 2-, originan el enfoque. Enfoque centrado, como afirman Shoemaker y Cohen (2006, p. 65), en la "relevancia pública de los hechos que afectan el bienestar de la ciudadanía".

En nuestro análisis hemos observado que términos clásicos como pirámide invertida o verdad tienden a excluirse del concepto medular de la objetividad. La pirámide invertida desaparece como forma de escribir la información (Armentía, Caminos, Elexgaray et al., 2000a; Oller y Barredo, 2012), por lo que se fosiliza también en lo ontológico. El concepto de verdad, tan apegado a soluciones ideológicas pasadas, se multiplica infinitamente en una sociedad que Flichy ha definido como la "sociedad del individualismo conectado" (García Galindo, 2009, p. 9). Al desaparecer esas posturas centrales, la objetividad del periodismo se conjuga como una interacción con el entorno, hacia la indagación constante, y la reconversión del 
profesional más como estructurador -o gatewatcher, según la aclaración introducida por Bruns (2003)-, o como gestor simbólico.

\section{Referencias}

Alexa, M. (1997). Computer-assisted text analysis methodology in the social sciences. En Zuma Arbeitsbericht, 97(07). Recuperado de http://www.gesis.org/fileadmin/upload/forschung/publikationen/gesis reihen/zuma arbeit sberichte/97 07.pdf

Armentía, J. I., Caminos, J. M., Eelxgaray, J., Marín, F. y Merchán, I. (2000). El Diario Digital. Análisis de los contenidos textuales, aspectos formales y publicitarios. Barcelona: Bosch. Recuperado http://books.google.com.pa/books/about/El diario digital.html?hl=es\&id=qshAOwAACAAJ

Barredo, D. (2013). Monarquía, consenso y democracia. Análisis de contenido informatizado de las coberturas sobre el rey Juan Carlos I en ABC.es y ELPAíS.com (2009 - 2011). Quito: CIESPAL.

Recuperado de http://www.publicacionesciespal.org/index.php/component/content/article?id=195:monarqu ia-consenso-y-democracia-analisis-de-contenido-informatizado-de-las-coberturas-sobreel-rey-juan-carlos-i-en-abc-es-y-elpais-com-2009-2011

Barredo Ibáñez, D. y Oller, M. (2012). Tratamiento de género en las informaciones ofrecidas sobre el Día Internacional de la Mujer Trabajadora (2001 - 2010) en ABC.es. En J. C. Suárez, I. Liberia y B. Zurbano, (Eds.), Libro de Actas del I Congreso Internacional de Comunicación y Género (pp. 520 - 540). Sevilla: Editorial Mad.

Barredo Ibáñez, D. y Oller, M. (2012). Género, ideología y prensa digital. La construcción informativa del Día Internacional de la Mujer Trabajadora en www.abc.es y www.elpais.com (2001 - 2010). España: Revista Mediterránea de Comunicación. Recuperado de http://www.rmedcom.org/libros/2Barreda-Oller.pdf

Bruns, A. (May, 2003). Gatewatching, not gatekeeping: Collaborative online news. The Uses of the Internet, 107, 31-44. Recuperado de http://www.uq.edu.au/mia/2003issues\#abstracts2

Buenaventura, S.; Barredo, D. y Oller, M. (2013). El sujeto AP genera identidades infinitas. Análisis de contenido informatizado de los Diarios de Alejandra Pizarnik. En A. De Chatellus y M. Ezquerro. (Eds.), Alejandra Pizarnik: el lugar donde todo sucede (pp. 243 268). París: L'Harmattan. Recuperado de http://www.editionsharmattan.fr/jeunesse/index.asp?navig=catalogue\&obj=livre \&no=41520

Canel, M. J. (1999). El País, ABC y El Mundo: tres manchetas, tres enfoques de las noticias. En Zer: Revista de Estudios de Comunicación, 6, 23-46. Recuperado de http://www.ehu.es/zer/zer6/5canel.htm

Daniel Barredo, Martín Oller y Sergio Hernández

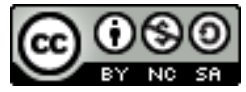

Esta obra está bajo licencia 
Chen, H., Evans, C., Battleson, B., Zubrow, E. \& Woelfel, J. (October, 2011). Procedures for the precise analysis of massive textual datasets. Communication \& Science Journal, 10.

Recuperado de http://www.galileoco.com/literature/chen11.pdf

Deuze, M. (2002). National News Cultures. A comparison of Dutch, German, British, Australian, and U.S. Journalism. Journalism and Mass Communication Quarterly, 79(1), p. 134-149. doi: http://dx.doi.org/10.1177/107769900207900110

Diefenbach, D. L. (2001). Historical Foundations of Computer-Assisted Content Analysis. En West, M. D. (Ed.), Theory, Method, and Practice in Computer Content Analysis (pp. 13 41). Westport, CT: Ablex Publishing. Recuperado de http://goo.gl/njkQXI

Donsbach, W. (1990). Objektivitätsmasse in der Publizistikwissenschaft. Publizistik. 36, 18-29.

Donsbach, W. \& Klett, B. (1993). Subjective - objectivity. How journalists in four countries define a key term of their profession. International Communication Gazette. 51(1), 53-83. Recuperado de http://dx.doi.org/10.1177/001654929305100104

García Galindo, J. A. (octubre-diciembre, 2009). La Sociedad de la Información (SI). Su historia a través de TELOS. Telos: Cuadernos de Comunicación e Innovación, 81. Recuperado de http://telos.fundaciontelefonica.com/seccion=1268\&idioma=es ES\&id=200911031247000 $\underline{1 \& \text { activo }=6 . \text { do }}$

Hackett, R. A. (2008). Objectivity in reporting. The International Encyclopedia of Communication. http://www.communicationencyclopedia.com/subscriber/tocnode?id=g9781405131995_ch unk_g978140513199520_ss1-1 doi: 10.1111/b.9781405131995.2008.x

Hanitzsch, T. (November, 2007). Deconstructing Journalism Culture: Toward a Universal Theory. Communication theory, 17(4), 367-385. doi: http://dx.doi.org/10.1111/j.14682885.2007.00303.x

Hernández, H. (2006). Neologismos, medios de comunicación y lexicografía. En Vilches Vivancos, F. (Ed.), Creación neológica y nuevas tecnologías (pp. 167-188). Madrid: Dykinson. Recuperado de http://www.dykinson.com/libros/creacion-neologica-y-nuevastecnologias/9788497726245/

Jarren, O. y Vogel, M. (2008). Gesellschaftliche Selbstbeobachtung und Ko-Orientierung. Die Leitmedien der modernen Gesellschaft. En Gendolla, P.; Ligensa, A. y Müller, D. (Eds.). Alte und neue Leitmedien (pp. 71-91). Bielefeld: Transcript Verlag.

Lippmann, W. (1920). Liberty and the news. New York: Harcourt, Brace \& Howe.

Daniel Barredo, Martín Oller y Sergio Hernández 
Mayring, P. (2002). Einführung in die qualitative Sozialforschung. Eine Anleitung zu qualitativem Denken ( $5^{\mathrm{a}}$ ed.). Weinheim, Basel: Beltz Verlag. Recuperado de http://goo.gl/pJcucU

Oller, M. y Meier, K. (2012). La cultura periodística de España y Suiza. Madrid: Fragua. Recuperado de http://goo.gl/OpOKb5

Oller, M. y Barredo, D. (2012). La Sociedad de los Ideantes: Repensando los conceptos de opinión y esfera pública y las teorías democráticas relacionadas con el fenómeno comunicativo ciudadano. España: Cuadernos Artesanos de la Latina. Recuperado de http://issuu.com/revistalatinadecomunicacion/docs/oller

Oller, M., Chavero, P. Ortega, E. y Pullas, T. (2014). La idea de objetividad de los periodistas en Ecuador. Index.Comunicación, 4(1): 237-262. Recuperado de http://journals.sfu.ca/indexcomunicacion/index.php/indexcomunicacion/article/view/160/15 $\underline{1}$

Popping, R. (2000). Computer-assisted text analysis. Londres: Sage. Recuperado de http://www.uk.sagepub.com/books/Book205979?siteld=sageuk\&prodTypes=any\&q=popping\&fs=1

Saad, A. (mayo-julio, 2012). La subjetividad en el discurso periodístico. Seguir creyendo en la objetividad como paradigma del periodismo es condenar al oficio a darle la espalda a la narración, la interpretación y la humanización de las historias, elementos que el público está reclamando. Razón y Palabra, $79 . \quad$ Recuperado de http://www.razonypalabra.org.mx/N/N79/V79/34 Saad V79.pdf

San Martín, R. (2008). Contra la objetividad. El mito de la neutralidad periodística y las alternativas para repensarlo. Perspectivas de la Comunicación, 1(2), 73-80. Recuperado de http://goo.gl/LUB7Bo

Schudson, M. (2001). The objetivity norm in American journalism. Journalism. 2, 149-170. doi: http://dx.doi.org/10.1177/146488490100200201

Shoemaker, P. y Cohen, A. (2006). News around the world. New York: Routledge. Recuperado de http://www.routledge.com/books/details/9780415975063/

Tian, Y. \& Stewart, C. M. (2005). Framing the SARS Crisis: A Computer-Assisted Text Analysis of CNN and BBC Online News Reports of SARS. Asian Journal of Communication. 15(3), p. 289-301. doi: http://dx.doi.org/10.1080/01292980500261605

Tuchman, G. (1978). Making news: A study in the construction of reality. New York: The Free Press. Recuperado de http://goo.gl/qPfd1v

Valbuena, F. (1997). Teoría General de la Información. Madrid: Editorial Noesis.

Woelfel, J. (1998) User's guide. CatPac II (Version 2.0). Recuperado de http://www.galileoco.com/Manuals/CATPAC3.pdf

Daniel Barredo, Martín Oller y Sergio Hernández

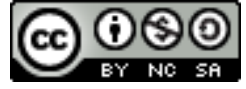

Esta obra está bajo licencia 


\section{Anexo I}

Dendrograma de las 40 palabras-tema más frecuentes en la entrevista sobre objetividad

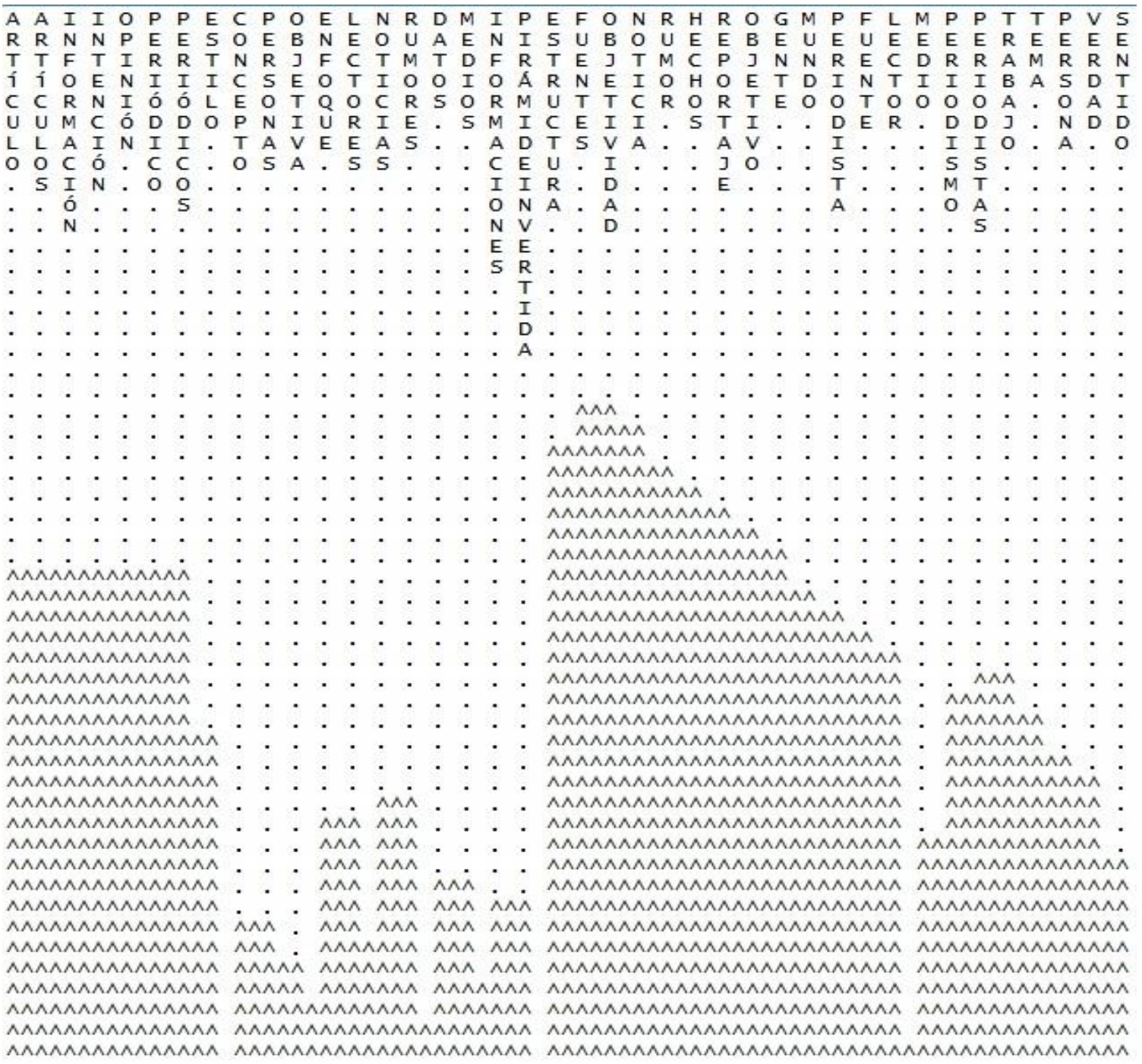

Fuente: Catpac / elaboración propia

Daniel Barredo, Martín Oller y Sergio Hernández

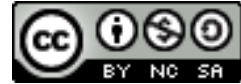

Esta obra está bajo licencia

Creative Commons Reconocimiento-NoComercial-Compartirlgual 4.0 Internacional License 